\title{
Expression of pigment epithelium-derived factor is associated with a good prognosis and is correlated with epithelial-mesenchymal transition-related genes in infiltrating ductal breast carcinoma
}

\author{
DAN ZHOU ${ }^{1,2^{*}}$, MIN ZHANG $^{3 *}$, PENGCHENG XU ${ }^{3}$, YANG YU $^{3}$, GUOLIN YE $^{2}$, LIN ZHANG $^{3}$ and AIGUO WU ${ }^{1}$ \\ ${ }^{1}$ Department of General Surgery, Zhujiang Hospital Affiliated to Southern Medical University, Guangzhou, \\ Guangdong 510282; ${ }^{2}$ Department of Breast Surgery, Foshan Hospital Affiliated to Sun Yat-Sen University, \\ The First People's Hospital of Foshan, Foshan, Guangdong 528100; ${ }^{3}$ Department of Histology and Embryology, \\ State Key Laboratory, Southern Medical University, Guangzhou, Guangdong 510515, P.R. China
}

Received September 25, 2014; Accepted June 11, 2015

DOI: $10.3892 / \mathrm{ol} .2015 .3880$

\begin{abstract}
Epithelial-mesenchymal transition (EMT) is a pivotal event in the progression of cancer towards metastasis. Given that pigment epithelium-derived factor (PEDF) inhibits angiogenesis, the present study analyzed whether PEDF expression is associated with EMT and prognosis in invasive ductal breast cancer (IDC). Immunohistochemical analysis was used to examine the expression levels of PEDF, E-cadherin, vimentin, Snail and nuclear factor $-\kappa \mathrm{B}(\mathrm{NF}-\kappa \mathrm{B})$ in 119 cases of IDC. Correlations between PEDF expression and EMT-related genes, and clinicopathological features and clinical prognosis were analyzed. E-cadherin, vimentin, Snail and NF- $\mathrm{NB}$ expression was correlated with tumor size, lymph node metastasis and clinicopathological stage. PEDF expression was closely associated with tumor size. Spearman's rank correlation analysis revealed a positive correlation between PEDF and E-cadherin, vimentin, Snail and NF- $\kappa$ B expression $(\mathrm{P}<0.05)$. Additionally, Kaplan-Meier survival analysis demonstrated that the five-year survival rate was higher for patients with PEDF- and E-cadherin-positive tumors, but was lower for those with vimentin-, Snail- and NF- $\kappa \mathrm{B}$-positive tumors. Vimentin, E-cadherin and NF- $\kappa \mathrm{B}$ levels were dependent prognostic factors of favorable outcomes in IDC, as
\end{abstract}

Correspondence to: Professor Aiguo Wu, Department of General Surgery, Zhujiang Hospital Affiliated to Southern Medical University, 253 Industrial Road, Haizhu, Guangzhou, Guangdong 510282, P.R. China

E-mail: 402337056@qq.com

Professor Lin Zhang, Department of Histology and Embryology, State Key Laboratory, Southern Medical University, 1838 Guangzhou Avenue, Baiyun, Guangzhou, Guangdong 510515, P.R. China

E-mail: zlilyzh@126.com

${ }^{*}$ Contributed equally

Key words: pigment epithelium-derived factor, breast cancer, epithelial-mesenchymal transition, prognosis determined by Cox multivariate analysis. PEDF expression in breast cancer was significantly associated with EMT-related genes, suggesting that it may be an EMT suppressor. However, its potential as a prognostic indicator in breast cancer warrants further investigation.

\section{Introduction}

As the most commonly diagnosed cancer and the main cause of cancer-related mortality among women, breast cancer accounts for $23 \%$ of all cancer cases and $14 \%$ of the cancer-related fatalities (1). Breast cancer is also one of the most common malignant tumors in Chinese women, with $>100$ new cases per 100,000 women aged 55-69 years estimated to occur by 2021 (2). Advances in the treatment of this disease using a multi-disciplinary approach with improved combinations of surgery, radiotherapy, chemotherapy and endocrine therapy have resulted in marked improvements in patient outcomes. However, more than half a million women will continue to succumb to breast cancer annually despite these advances (3).

Epithelial-mesenchymal transition (EMT) is an essential process during cancer progression to tumor metastasis $(4,5)$. Mesenchymal cell marker expression, such as that of vimentin, Snail $(6,7)$ and E-cadherin (8), is considered a hallmark for EMT. EMT can also be induced in mammary epithelial cells by expression of various factors, such as the Twist or Snail families $(9,10)$. In addition, the upregulation of nuclear factor- $\kappa B$ $(\mathrm{NF}-\kappa \mathrm{B})$ is found in human breast tumor cell lines, carcinogen-transformed mammary epithelial cells, and the majority of primary human and rodent breast tumor tissue samples (11). $\mathrm{NF}-\kappa \mathrm{B}$ has also been shown to be a central mediator of EMT in a breast cancer progression mouse model $(12,13)$. Although activation of these processes in breast cancer cells has greatly increased their invasive and metastatic potential, the exact role of EMT in tumor metastasis remains unknown (14).

Pigment epithelium-derived factor (PEDF) is a potential independent prognostic marker for breast cancer, and reduction in its expression levels is associated with the progression of disease and a poor patient outcome $(15,16)$. In addition to its anti-angiogenic functions, PEDF also inhibits tumor cell 
migration by promoting cell adhesion, inducing apoptosis and regulating tumor cell differentiation (17). Specifically, PEDF induces endothelial cell apoptosis via NF- $\kappa$ B activation and its downstream target Fas ligand $(18,19)$, Furthermore, PEDF expression is significantly reduced in a wide range of tumor types, and its recovered expression in these tumors delays the onset of primary tumors and decreases metastasis (20). In the brain, PEDF acts as a metastatic suppressor and a neuroprotectant, highlighting its role in limiting brain metastasis and local consequences in primary breast tumors (21). Thus, PEDF may play a critical role in breast cancer development and progression. However, the exact molecular mechanisms by which PEDF elicits its antitumor effects remain unknown (22). The effects of PEDF on tumor suppression and endothelial cell apoptosis combined with its inhibition of tumor cell migration suggest that it may have therapeutic value in the context of EMT. The present study systemically investigated the association between PEDF levels and EMT-related proteins in 119 cases of primary invasive ductal breast cancer (IDC), and analyzed their correlation with clinicopathological factors and patient survival to determine the association between PEDF expression and EMT in breast cancer.

\section{Materials and methods}

Patients and tissue specimens. Paraffin-embedded surgical specimens were randomly obtained from 119 non-consecutive breast cancer patients that underwent modified radical masectomy at the Zhujiang Hospital Affiliated to Southern Medical University (Guangzhou, Guangdong, China) between 2006 and 2008. The 119 surgical specimens were selected in accordance with the following criteria: Female patients presenting with unilateral, primary IDC without a history of breast cancer. Patients who received neoadjuvant chemotherapy prior to surgery, presented with secondary breast cancer or exhibited peritumorous carcinoma in situ in the tumor sample were excluded. Tumor histology was determined according to the 2003 World Health Organization criteria (23), while disease stage was assessed according to the Union for International Cancer Control (24). Tumors were graded according to Bloom and Richardson, as modified by Elston and Ellis (25), and hormone receptor status was assessed according to the scoring system developed by Remmele and Stegner (26). Inclusion criteria for the study were as follows: Female patients presenting with unilateral, primary IDC, without a history of breast cancer. Patients who received neo-adjuvant chemotherapy prior to surgery, presented with secondary breast cancer or had peritumorous carcinoma in situ present in the tumor sample were excluded. Normal mammary parenchyma obtained from 30 women who underwent breast reduction was also analyzed. Ethical approval was obtained from the Medical Ethics Committee of Zhujiang Hospital Affiliated to Southern Medical University and written informed consent was obtained from all patients.

Immunohistochemical staining. Paraffin-embedded sections (5- $\mu \mathrm{m}$ thick) were deparaffinized by immersion in dimethylbenzene for $20 \mathrm{~min}$ and then rehydrated in graded concentrations of ethanol (100, 90, 80 and 70\%; Beyotime
Biotechnology, Haimen, China). The sections were then subjected to immunohistochemical analysis, as previously described by Zhang et al (27). Subsequent to blocking endogenous peroxidase (3\% hydrogen peroxidase; Beyotime Biotechnology), the sections were incubated with primary mouse anti-human monoclonal PEDF (1:100; Millipore, Billerica, MA, USA), rabbit anti-human monoclonal E-cadherin (1:500; Millipore), mouse anti-human monoclonal vimentin (1:100; Cell Signaling Technology Inc., Danvers, MA, USA), goat anti-human polyclonal Snail (1:50; Santa Cruz Biotechnology Inc., Dallas, TX, USA) and rabbit anti-human monoclonal NF- $\kappa$ B (1:600; Cell Signaling Technology Inc.) antibodies diluted in phosphate-buffered saline containing $0.1 \%$ Tween-20 (PBST) and 5\% bovine serum albumin (Beyotime Biotechnology) overnight at $4^{\circ} \mathrm{C}$. Subsequent to being washed three times with PBST, the sections were incubated with secondary antibodies (goat anti-mouse IgG/biotin, rabbit anti-goat $\mathrm{IgG} /$ biotin or goat anti-rabbit IgG/biotin; 1:100), avidin-biotin-peroxidase complex and DAB reagent (Wuhan Boster Biological Technology, Ltd., Wuhan, China). Subsequently, all sections were counterstained with hematoxylin (Beyotime Biotechnology) and visualized by microscopy (DM40008; Leica, Solms, Germany). Images were captured by Leica Application Suite 3.7 (Leica), and 5-10 photomicrographs were randomly selected from each section.

Immunohistochemical evaluation. The expression levels of PEDF, E-cadherin, vimentin, Snail and NF- $\kappa$ B were independently reviewed and scored by two pathologists who were blinded to the clinical parameters. The expression of Snail and NF- $\mathrm{NB}$ was observed in the cytoplasm, nucleus or both; however, only nuclear expression was considered immunopositive for Snail. Expression of PEDF, E-cadherin and vimentin in the cytoplasm and/or plasma membrane were each considered positive.

The semi-quantitative analysis of the distribution of staining was scored according to the percentage of cells showing immunoreactivity: Negative immunoreactivity indicated the absence of staining or weak staining in $1 \%$ of the tumor cells; + indicated focal staining in 1-10\% of the tumor cells; ++ indicated positive staining in $11-50 \%$ of the tumor cells; and +++ indicated positive staining in $>50 \%$ of the tumor cells. Tumors were defined as immunopositive when $>10 \%(++/+++)$ of tumor cells show immunoreactivity. Thus, $(+)$ is defined as low expression, whereas $(++/+++)$ is defined as high expression.

Statistical analysis. SPSS version 13.0 (SPSS, Inc., Chicago, IL, USA) was used for all statistical analyses. The $\chi^{2}$ test was used to analyze the correlation between PEDF, E-cadherin, vimentin, Snail and NF- $\kappa$ B expression, and the clinicopathological features of the IDC patients. Spearman's correlation coefficient analysis was used to evaluate the correlations between the variables. The Kaplan-Meier method and log-rank tests were used to evaluate the correlation between marker expression and overall survival (OS). The Cox proportional hazards model was used for the multivariate analysis to identify independent prognostic factors. $\mathrm{P}<0.05$ was considered to indicate a statistically significant difference. 


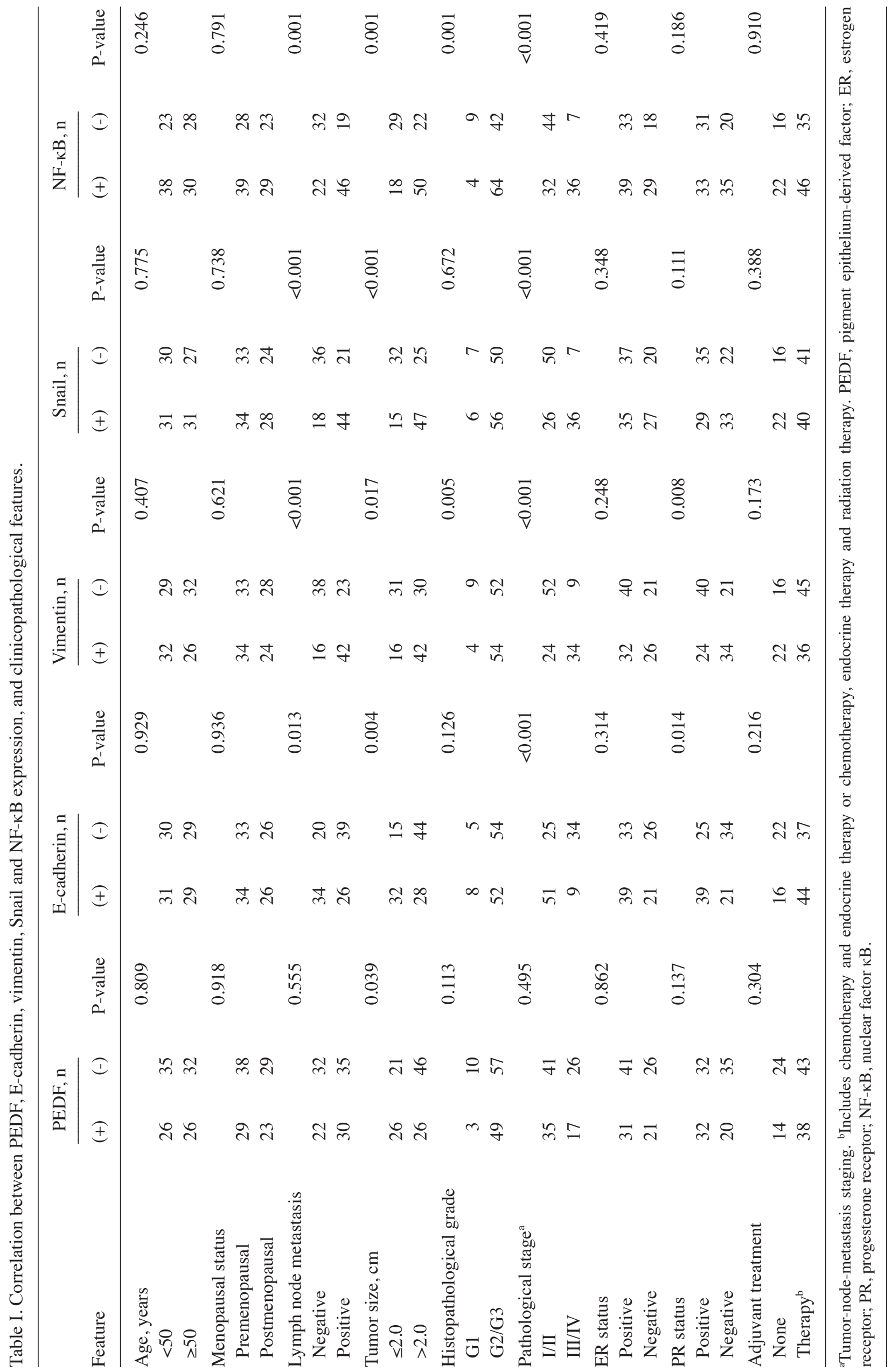




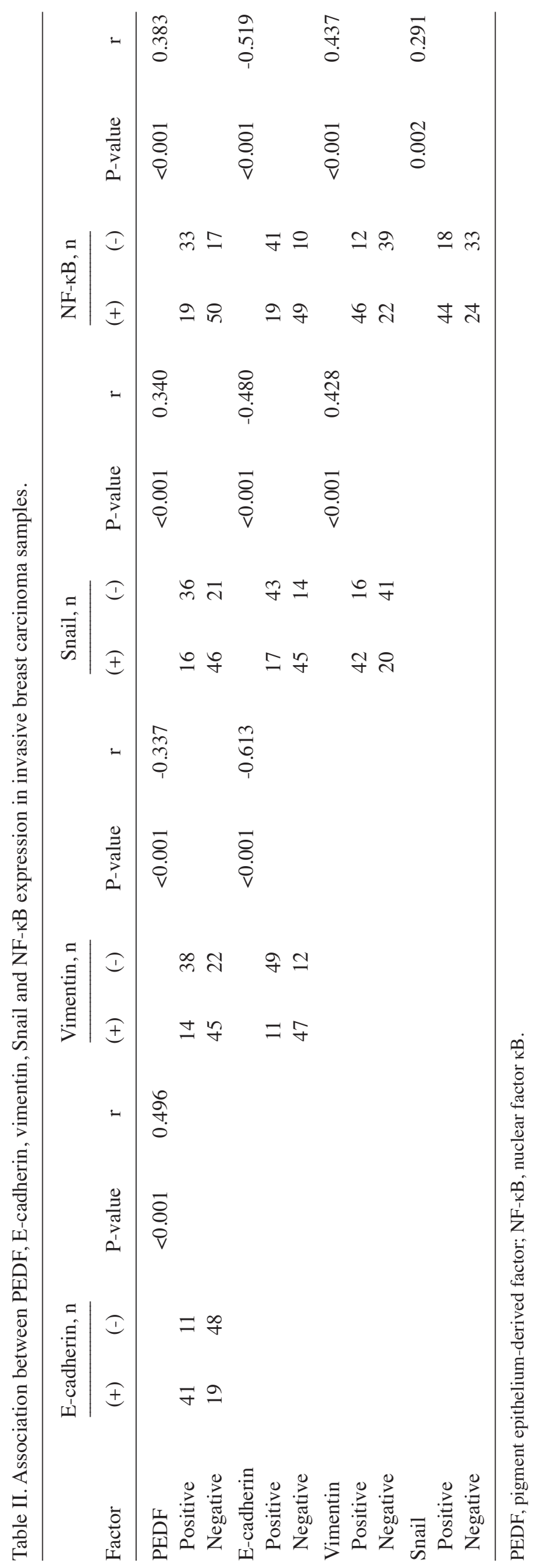

\section{Results}

PEDF, E-cadherin, vimentin, Snail and $N F-\kappa B$ expression in IDC samples. The expression of PEDF, E-cadherin, vimentin, Snail and NF- $\mathrm{KB}$ was analyzed in 119 IDC tissues by immunohistochemistry (Fig. 1). As shown in Fig. 1A, PEDF was detected in the cytoplasm of the epithelial cells, and high levels of staining were observed in $44.5 \%$ of the breast carcinoma tissues analyzed, which were classified as PEDF-positive. Although E-cadherin was present in the cell membranes of normal breast tissues, it was absent in the tumor tissues (Fig. 1G). Approximately 49.6\% (59/119) of the tumor sections exhibited an absence or reduction in E-cadherin expression (Table I). Reduced E-cadherin expression was observed in $20.9 \%$ (9/43) of the late-stage (III/IV) and $49.1 \%(52 / 106)$ of the high-grade tumors, which was significantly more than that observed for the early-stage (I/II; 67.1\%) and low-grade (61.5\%) tumors (both $\mathrm{P}=0.001$ ) (Table I). Conversely, vimentin and Snail were absent in the normal breast tissues (Fig. 1C and E).

However, vimentin was highly expressed in the tumor tissues, and Snail was detected in $52.1 \%$ of the tumor tissues analyzed (Fig. $1 \mathrm{H}$ and J; Table I). Finally, NF- $\kappa \mathrm{B}$ was detected in the cytoplasm of IDC cells in $57.1 \%$ of the tumors analyzed (Fig. 1I).

Correlation between PEDF, E-cadherin, vimentin, Snail and $N F-\kappa B$ expression, and clinicopathological features. The correlation analysis between the expression levels of PEDF, E-cadherin, vimentin, Snail, NF- $\kappa B$ and clinicopathological features is summarized in Table I. PEDF protein was detected in $40.7 \%$ of lymph node-negative tumors and $46.2 \%$ of lymph node-positive tumors (Table I). The cytoplasmic expression of PEDF was significantly correlated with the tumor size $(\mathrm{P}=0.039)$. Furthermore, a low level of $\mathrm{E}$-cadherin expression was correlated with a large tumor size $(\mathrm{P}=0.004)$, positive lymph node metastasis status $(\mathrm{P}=0.013)$, early pathological stage $(\mathrm{P}<0.001)$ and positive progesterone receptor (PR) status $(\mathrm{P}=0.014$; Table $\mathrm{I})$. High vimentin expression was strongly associated with high pathological stage and large tumor size $(\mathrm{P}<0.001$ and $\mathrm{P}=0.009$, respectively); it was also correlated with positive lymph node status and negative PR status ( $\mathrm{P}<0.001$ and $\mathrm{P}=0.008$, respectively) (Table I). High nuclear Snail staining was significantly associated with a large tumor size $(\mathrm{P}<0.001)$, positive lymph node metastasis status $(\mathrm{P}<0.001)$ and late pathological stage $(\mathrm{P}=0.001)$. For example, $83.7 \%$ of patients with late-stage tumors (III-IV) expressed high levels of nuclear Snail, compared with 26.3\% of patients with early-stage (I-II) tumors. Positive nuclear expression of NF- $\mathrm{\kappa B}$ was also associated with all adverse clinicopathological variables, namely tumor size, high tumor grade, late tumor stage and lymph node positivity (all $\mathrm{P} \leq 0.001)$ (Table I). No significant association existed between PEDF, E-cadherin, vimentin, Snail, NF- $\kappa B$ expression and gender, age, menopausal status, adjuvant treatment and estrogen receptor (ER) status.

Association between PEDF, E-cadherin, vimentin, Snail and $N F-\kappa B$ expression in IDC samples. Next, the correlation between low level PEDF expression levels and EMT-related 
Table III. Correlation of PEDF, E-cadherin, vimentin, Snail and NF- $\kappa$ B expression with patient survival.

\begin{tabular}{|c|c|c|c|c|c|c|}
\hline Factor & No. of cases & Positive, $\mathrm{n}$ & Negative, $\mathrm{n}$ & Score range & Median & P-value \\
\hline \multicolumn{7}{|l|}{ PEDF } \\
\hline NPI & & & & & & 0.701 \\
\hline 1 & 24 & 9 & 15 & $2.2-4.4$ & 3.3 & \\
\hline 2 & 52 & 24 & 28 & $2.6-6.4$ & 4.4 & \\
\hline 3 & 43 & 19 & 24 & $3.5-7.2$ & 5.8 & \\
\hline Survival status & & & & & & 0.014 \\
\hline Good & 49 & 28 & 21 & & & \\
\hline Poor & 70 & 24 & 46 & & & \\
\hline \multicolumn{7}{|l|}{ E-cadherin } \\
\hline NPI & & & & & & $<0.001$ \\
\hline 1 & 24 & 20 & 4 & $2.2-4.4$ & 3.3 & \\
\hline 2 & 52 & 29 & 23 & $2.6-6.4$ & 4.4 & \\
\hline 3 & 43 & 11 & 32 & $3.5-7.2$ & 5.8 & \\
\hline Survival status & & & & & & $<0.001$ \\
\hline Good & 49 & 39 & 10 & & & \\
\hline Poor & 70 & 21 & 49 & & & \\
\hline \multicolumn{7}{|l|}{ Vimentin } \\
\hline NPI & & & & & & $<0.001$ \\
\hline 1 & 24 & 5 & 19 & $2.2-4.4$ & 3.3 & \\
\hline 2 & 52 & 20 & 32 & $2.6-6.4$ & 4.4 & \\
\hline 3 & 43 & 33 & 10 & $3.5-7.2$ & 5.8 & \\
\hline Survival status & & & & & & $<0.001$ \\
\hline Good & 49 & 9 & 40 & & & \\
\hline Poor & 70 & 49 & 21 & & & \\
\hline \multicolumn{7}{|l|}{ Snail } \\
\hline NPI & & & & & & $<0.001$ \\
\hline 1 & 24 & 6 & 18 & $2.2-4.4$ & 3.3 & \\
\hline 2 & 52 & 24 & 28 & $2.6-6.4$ & 4.4 & \\
\hline 3 & 43 & 32 & 11 & $3.5-7.2$ & 5.8 & \\
\hline Survival status & & & & & & 0.005 \\
\hline Good & 49 & 18 & 31 & & & \\
\hline Poor & 70 & 44 & 26 & & & \\
\hline \multicolumn{7}{|l|}{$\mathrm{NF}-\kappa \mathrm{B}$} \\
\hline NPI & & & & & & $<0.001$ \\
\hline 1 & 24 & 5 & 19 & $2.2-4.4$ & 3.3 & \\
\hline 2 & 52 & 24 & 28 & $2.6-6.4$ & 4.4 & \\
\hline 3 & 43 & 39 & 4 & $3.5-7.2$ & 5.8 & \\
\hline Survival status & & & & & & $<0.001$ \\
\hline Good & 49 & 16 & 33 & & & \\
\hline Poor & 70 & 52 & 18 & & & \\
\hline
\end{tabular}

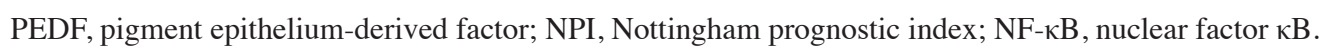

proteins was analyzed in the breast carcinoma tissues. In accordance with the protein changes found during EMT, high expression levels of vimentin, Snail and NF- $\kappa \mathrm{B}$ were associated with the weak expression of membranous E-cadherin $(\mathrm{P}<0.001$; Table II). In addition, a high level of vimentin expression was significantly correlated with low level nuclear Snail expression $(\mathrm{P}<0.001, \mathrm{r}=0.428)$; a high level of Snail expression was also significantly correlated with the low level expression of $\mathrm{NF}-\kappa \mathrm{B}$ $(\mathrm{P}=0.002, \mathrm{r}=0.291)$. These data indicated the presence of EMT in the IDC tissues. Furthermore, a high level of PEDF expression 

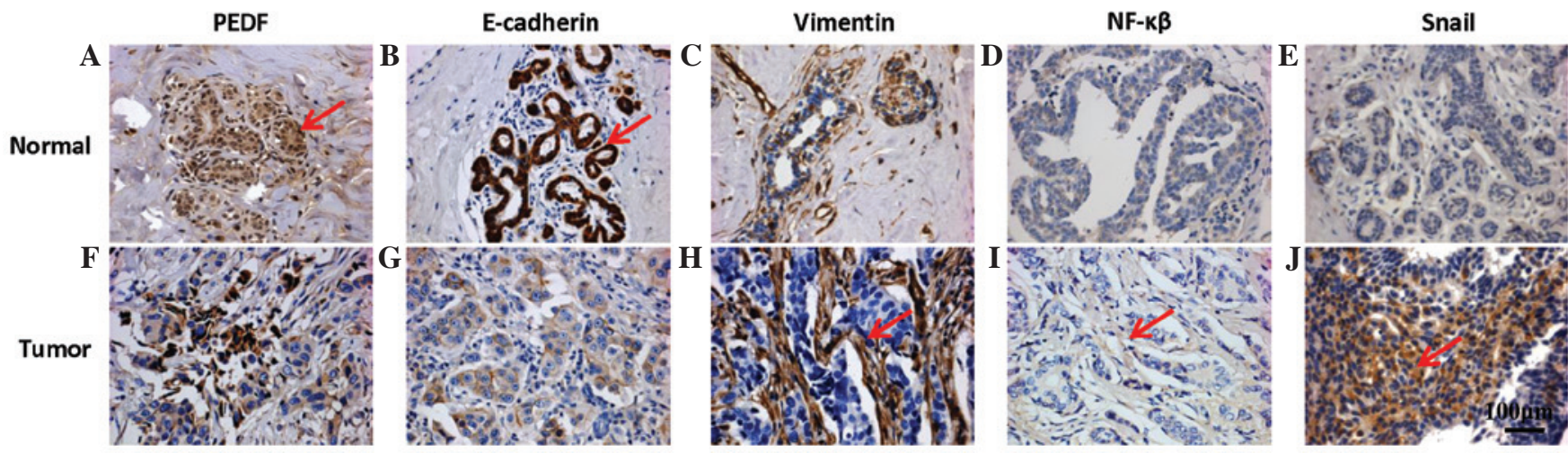

Figure 1. Immunohistochemical analysis of E-cadherin, vimentin, Snail and NF-kB in invasive ductal breast cancer (IDC) and normal breast samples (original magnification, x400). (A) Immunostaining of pigment epithelium-derived factor (PEDF) mainly in the cytoplasm of certain epithelial cells (yellow-brown granules indicated by the red arrow). (B)Immunostaining of E-cadherin in the cell membranes of normal breast tissues (yellow-brown granules indicated by the red arrow). (C) Immunostaining of vimentin in the normal breast tissues. (D) Immunostaining of nuclear factor $\kappa \beta$ (NF- $\kappa \beta$ ) in the normal breast tissues. (E) Immunostaining of Snail in the normal breast tissues.(F) Immunostaining of PEDF in invasive ductal breast cancer samples. (G) Immunostaining of E-cadherin in invasive ductal breast cancer samples. (H) Immunostaining of vimentin in the cytoplasm of certain epithelial cells (yellow-brown granules indicated by the red arrow). (I) Immunostaining of NF- $\mathrm{\beta} \beta$ in the nuclei and cytoplasm of cancer cells in a positive specimen (yellow-brown granules indicated by a red arrow). (J) Immunostaining of Snail in the cytoplasm of IDC cells in a positive specimen (yellow-brown granules indicated by a red arrow).
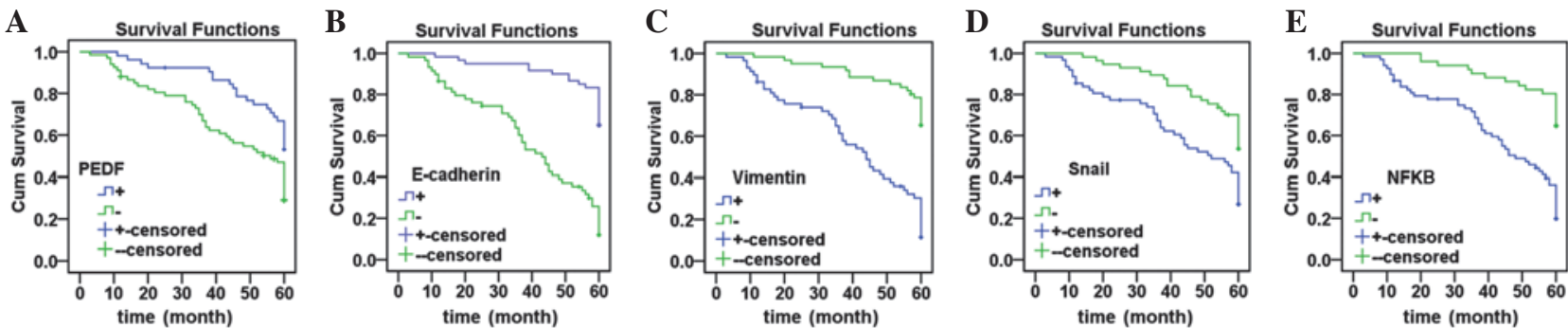

Figure 2. Kaplan-Meier analysis for overall survival curves of breast cancer patients with pigment epithelium-derived factor (PEDF), E-cadherin, vimentin, Snail and nuclear factor $\kappa \mathrm{B}(\mathrm{NF}-\mathrm{\kappa B})$ expression. Survival curves are stratified by negative (-) and positive (+) (A) PEDF, (B) E-cadherin, (C) vimentin, (D) Snail and (E) NF- $\kappa$ B expression.
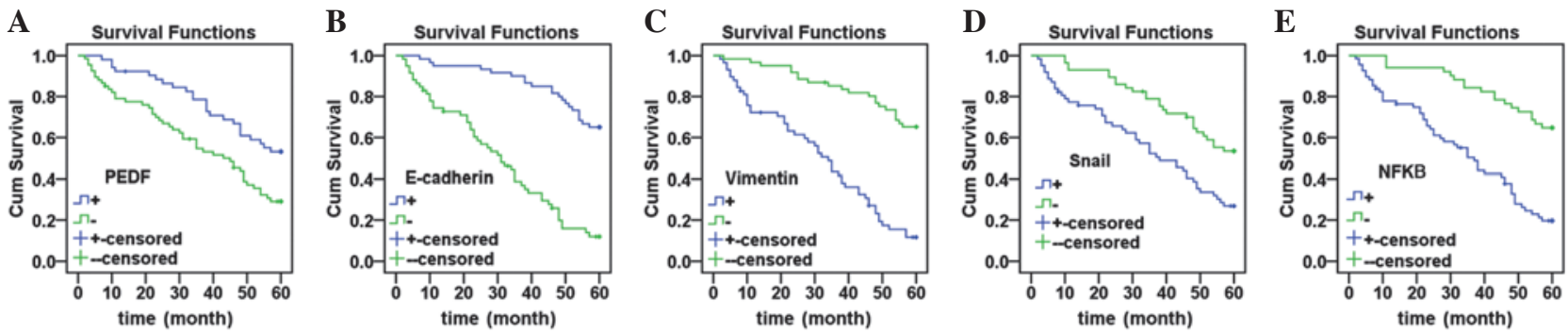

Figure 3. Kaplan-Meier analysis for disease-free survival curves of breast cancer patients with pigment epithelium-derived factor (PEDF), E-cadherin, vimentin, Snail and nuclear factor $\kappa B$ (NF-kB) expression. Survival curves are stratified by negative (-) and positive (+) (A) PEDF, (B) E-cadherin, (C) vimentin, (D) Snail and (E) NF- $\mathrm{kB}$ expression.

was strongly associated with a low level of E-cadherin expression $(\mathrm{P}<0.001, r=0.496)$ and a high level of vimentin $(\mathrm{P}<0.001$, $r=-0.337)$, Snail $(\mathrm{P}<0.001, r=0.34)$ and NF-kB $(\mathrm{P}<0.001$, $r=0.383$ ) expression. These data suggest that PEDF may be involved in the regulation of EMT.

Association between PEDF, E-cadherin, vimentin, Snail and $N F-\kappa B$ expression, and patient survival. Next, the Nottingham prognostic index (NPI) was used as an indicator of patient prognosis, as previously described (28). As shown in Table III, the overexpression of E-cadherin, vimentin, Snail and NF- $\mathrm{KB}$ was significantly associated with NPI status, as determined by the Kruskal-Wallis test (all $\mathrm{P}<0.001$ ); however, PEDF expression was not associated with NPI. Specifically, high NF-кB expression was more frequently observed in patients with a high NPI $(\geq 5.4 ; 37.7 \%)$ compared with those with a low NPI $(<5.4 ; 24.4 \%)$. The patients were then divided into two groups on the basis of their prognosis. Patients were considered to have a good prognosis $(n=49)$ if they remained disease-free at the 5 -year follow-up; those with a poor prognosis $(n=70)$ included patients who developed recurrence, metastasis to a distant site, or those who had succumbed as a result of the breast cancer. As 
Table IV. Multivariate analysis of overall survival of patients with invasive breast carcinoma.

\begin{tabular}{|c|c|c|c|c|c|c|}
\hline Factor & $\begin{array}{l}\text { Regression } \\
\text { coefficient }\end{array}$ & $\begin{array}{l}\text { Standard } \\
\text { error }\end{array}$ & Wald & $\mathrm{RR}$ & $95 \% \mathrm{CI}$ & P-value \\
\hline Age & -0.001 & 0.012 & 0.010 & 0.999 & $0.975-1.023$ & 0.921 \\
\hline Histopathological grading & 0.472 & 0.516 & 0.838 & 1.604 & $0.583-4.410$ & 0.360 \\
\hline Lymph node metastasis & 0.055 & 0.384 & 0.020 & 1.056 & $0.498-2.240$ & 0.886 \\
\hline Pathological stage & 0.735 & 0.505 & 2.120 & 1.329 & $0.775-5.614$ & 0.145 \\
\hline Tumor size & 0.285 & 0.109 & 6.853 & 2.086 & $1.074-1.645$ & 0.009 \\
\hline ER status & 0.399 & 0.263 & 2.298 & 1.49 & $0.890-2.497$ & 0.130 \\
\hline PR status & 0.368 & 0.270 & 1.844 & 1.445 & $0.849-2.459$ & 0.174 \\
\hline PEDF & -0.232 & 0.351 & 0.435 & 0.793 & $0.398-1.579$ & 0.510 \\
\hline Vimentin & -0.847 & 0.353 & 5.751 & 0.429 & $0.215-0.857$ & 0.016 \\
\hline E-caherin & 1.186 & 0.406 & 8.517 & 3.274 & $1.476-7.262$ & 0.004 \\
\hline Snail & -0.001 & 0.328 & 0.000 & 0.999 & $0.525-1.900$ & 0.997 \\
\hline $\mathrm{NF}-\kappa \mathrm{B}$ & -0.609 & 0.364 & 2.796 & 0.544 & $0.267-1.110$ & 0.094 \\
\hline
\end{tabular}

ER, estrogen receptor; PR, progesterone receptor; PEDF, pigment epithelium-derived factor; RR, relative risk; $\mathrm{CI}$, confidence interval; NF- $\mathrm{kB}$, nuclear factor $\mathrm{\kappa B}$.

Table V. Multivariate analysis of disease-free survival of patients with invasive breast carcinoma.

\begin{tabular}{|c|c|c|c|c|c|c|}
\hline Factor & $\begin{array}{l}\text { Regression } \\
\text { coefficient }\end{array}$ & $\begin{array}{l}\text { Standard } \\
\text { error }\end{array}$ & Wald & RR & $95 \% \mathrm{CI}$ & P-value \\
\hline Age & -0.001 & 0.013 & 0.003 & 0.999 & $0.975-1.025$ & 0.959 \\
\hline Histopathological grading & 0.309 & 0.510 & 0.368 & 1.362 & $0.501-3.702$ & 0.544 \\
\hline Lymph node metastasis & -0.201 & 0.380 & 0.281 & 0.818 & $0.388-1.723$ & 0.596 \\
\hline Pathological stage & 0.528 & 0.505 & 1.095 & 1.696 & $0.631-4.563$ & 0.295 \\
\hline Tumor size & 0.219 & 0.108 & 4.134 & 1.245 & $1.008-1.537$ & 0.042 \\
\hline ER status & 0.404 & 0.268 & 2.270 & 1.497 & $0.886-2.531$ & 0.132 \\
\hline PR status & 0.257 & 0.270 & 0.907 & 1.294 & $0.762-2.197$ & 0.341 \\
\hline PEDF & -0.324 & 0.354 & 0.840 & 0.723 & $0.361-1.446$ & 0.034 \\
\hline Vimentin & -0.918 & 0.366 & 6.301 & 0.399 & $0.195-0.818$ & 0.012 \\
\hline E-caherin & 1.141 & 0.407 & 7.847 & 3.129 & $1.409-6.950$ & 0.005 \\
\hline Snail & -0.102 & 0.319 & 0.101 & 0.903 & $0.483-1.690$ & 0.751 \\
\hline $\mathrm{NF}-\kappa \mathrm{B}$ & -0.705 & 0.362 & 3.787 & 0.494 & $0.243-1.005$ & 0.052 \\
\hline
\end{tabular}

ER, estrogen receptor; PR, progesterone receptor; PEDF, pigment epithelium-derived factor; $\mathrm{RR}$, relative risk; $\mathrm{CI}$, confidence interval; $\mathrm{NF}-\mathrm{\kappa} B$, nuclear factor $\kappa \mathrm{B}$.

shown in Table III, patients with poor prognoses had low levels of PEDF and E-cadherin expression $(\mathrm{P}=0.014$ and $\mathrm{P}<0.001$, respectively) and high levels of vimentin, Snail and $\mathrm{NF}-\kappa \mathrm{B}$ expression $(\mathrm{P} \leq 0.005)$.

Univariate and multivariate Cox regression analysis of $P E D F, E$-cadherin, vimentin, Snail and $N F-\kappa B$ expression, and clinicopathological variables. Univariate analyses of OS using Cox regression analysis identified PEDF, vimentin, E-cadherin, Snail and NF- $\kappa \mathrm{B}$ expression $(\mathrm{P}=0.006, \mathrm{P}<0.001$, $\mathrm{P}<0.001, \mathrm{P}=0.001$ and $\mathrm{P}<0.001$, respectively), lymph node metastasis $(\mathrm{P}=0.015)$, tumor size $(\mathrm{P}=0.012)$, pathological stage $(\mathrm{P}=0.012)$ and $\mathrm{PR}$ status $(\mathrm{P}=0.003)$ as significant prognostic predicators (Table IV). To determine whether PEDF-positive expression was an independent predictor of patient survival, a multivariate analysis was performed using Cox proportional regression models, together with vimentin, E-cadherin, Snail and $\mathrm{NF}-\kappa \mathrm{B}$ expression, as well as basic patient and tumor characteristics, such as age, tumor clinical stage, lymph node metastasis, tumor size and ER/PR status. Cox multivariate analysis showed that the expression of vimentin and E-cadherin were independent prognostic factors associated with OS $(\mathrm{P}=0.016$ and $\mathrm{P}=0.004$, respectively) and disease-free survival (DFS; $\mathrm{P}=0.012$ and $\mathrm{P}=0.005$, respectively). Although PEDF expression was not correlated with $\mathrm{OS}(\mathrm{P}=0.51)$, a significant correlation with DFS was noted $(\mathrm{P}=0.034)$. With the exception 
of tumor size $(\mathrm{P}=0.009)$, no clinicopathological factors were independently predictive of patient survival (Table V).

Kaplan-Meier survival analysis. Kaplan-Meier survival curves are shown in Figs. 2 and 3. Among the 119 patients with IDC analyzed, the patients with PEDF- and E-cadherin-positive tumors exhibited higher OS rates compared with those with PEDF- and E-cadherin-negative tumors $(\mathrm{P}=0.004$ and $\mathrm{P}<0.001$, respectively; Fig. 2A and B). Patients with PEDF- and E-cadherin-positive tumors also exhibited significantly higher DFS rates ( $\mathrm{P}=0.004$ and $\mathrm{P}<0.001$, respectively; Fig. $3 \mathrm{~A}$ and $\mathrm{B})$. By contrast, the patients with vimentin-, Snail- and NF- $\kappa B-$ positive tumors exhibited lower OS rates compared with the patients with vimentin-, Snail- and NF- $\mathrm{NB}$-negative tumors (all $\mathrm{P} \leq 0.001$; Fig. 2C-E). Patients with vimentin-, Snail- and NF-кB-positive tumors also exhibited significantly lower DFS rates (all $\mathrm{P} \leq 0.001$; Fig. 3C-E).

\section{Discussion}

PEDF is a $50-\mathrm{kDa}$ protein found in the extracellular matrix (ECM). It belongs to the serpin (serine protease inhibitor) family, and contains heparin and collagen binding sites (29-31). PEDF is a potent neurotrophic and angiogenesis-inhibiting factor with tumor suppressor properties (32). In vitro studies have demonstrated that the silencing of PEDF may be a novel mechanism for the development of endocrine resistance in breast cancer and that its expression may be a predictive marker of endocrine sensitivity (33). In addition, previous studies have shown that the expression of PEDF is significantly decreased in a number of tumor types, including pancreatic adenocarcinoma (34), glioblastoma (35) and ovarian carcinoma (36).

Vascular endothelial growth factor receptor-1 (VEGFR-1) regulates EMT for the promotion of breast cancer progression and metastasis (37). In various types of tumor cells, PEDF has been shown to decrease VEGF levels (38-40); suppression of VEGF signaling by PEDF may be a novel therapeutic target. In addition, Cai et al (41) also identified two novel pathways through which VEGF-induced angiogenesis is inhibited by PEDF: Regulated intramembrane proteolysis and phosphorylation inhibition. Therefore, we hypothesized that PEDF may represent a potential biomarker of EMT in breast cancer and analyzed its expression using immunohistochemistry analysis. In the present study, high PEDF expression was strongly associated with low E-cadherin expression, as well as with high vimentin, Snail and NF-kB expression. These data suggest that PEDF may be involved in the regulation of EMT. This is the first study to show that PEDF may serve as a novel EMT suppressor and to reveal its potential as a prognostic indicator in breast cancer.

EMT exhibits certain characteristic phenotypic changes that are a result of complex genetic changes, which to a certain degree are mediated by specific transcription factors that are able to modulate E-cadherin expression and the expression of numerous other EMT-associated genes in vitro. Vimentin is a widely recognized EMT-like phenotype marker, and its expression has been shown in a number of aggressive breast cancer cell lines (42). In the present study, low E-cadherin expression was associated with increased tumor size $(\mathrm{P}=0.004)$, lymph node metastasis status $(\mathrm{P}=0.013)$ and the pathological stage
$(\mathrm{P}<0.001)$. In addition, high vimentin expression was strongly associated with high-grade and late-stage tumors $(\mathrm{P}<0.001)$, which is consistent with the results reported by Lee et al (43). Notably, vimentin overexpression and reduced E-cadherin expression were significantly correlated with reduced survival and were independent predictors in multivariate analysis. Moreover, multivariate analysis showed that the expression of vimentin and E-cadherin were independent prognostic factors correlated with shorter OS and DFS times.

Several studies have demonstrated the expression of Snail at the tumor-stroma interface and in invasive breast cancers (44). As expected, high nuclear Snail staining was significantly associated with large tumor size $(\mathrm{P}<0.001)$, status of lymph node metastasis $(\mathrm{P}<0.001)$ and pathological stage $(\mathrm{P}=0.001)$ in the present study. Moreover, it was observed that positive nuclear NF- $\mathrm{kB}$ expression was associated with all adverse clinicopathological variables analyzed, including large tumor size, high tumor grade, late tumor stage and lymph node positivity, confirming its significance in the development and progression of cancer. Furthermore, low E-cadherin expression, and high vimentin, Snail and NF- $\mathrm{BB}$ expression levels were associated with shorter OS and DFS times, which is consistent with studies of other cancers $(45,46)$. Taken together, these results suggest that the expression of certain transcription factors, including NF- $\kappa \mathrm{B}$ and Snail, is associated with a poor prognosis in a range of different human cancer types (47).

Previous studies have reported that low PEDF expression is associated with angiogenesis in breast cancer (16). The present study also demonstrated that PEDF was a statistically significant prognostic factor in multivariate Cox regression analysis. Additionally, although the association between EMT and metastasis in patients has been indicated, the present study is the first to suggest a possible mechanism by which PEDF may be capable of reversing tumor growth and metastasis in breast cancer. To the best of our knowledge, studies have rarely been focused on the PEDF and EMT-related genes; Hirsch et al (48) concluded that PEDF upregulates PPAR $\gamma$ by binding to PEDF receptor, resulting in the suppression of NF- $\mathrm{KB}$-mediated transcriptional activation in prostate cancer cells. Therefore, further studies are required to elucidate the mechanisms by which PEDF regulates EMT in breast cancer. Therapies targeting PEDF may provide a novel therapeutic approach for untreatable patients.

In summary, to the best of our knowledge, the present study is the first to demonstrate a link between the expression of PEDF in breast cancer and EMT-related genes. These findings suggest that PEDF may be capable of reversing tumor growth and metastasis in breast cancer; however, further studies are necessary to elucidate the association of PEDF expression and EMT in vitro and in vitro.

\section{Acknowledgements}

This study was supported by grants from the Natural Science Foundation of China (nos. 81171824, 81371719 and 81402613), the Natural Science Foundation of Guangdong province (nos. S2012010009276, 2014A030310055 and 2014A030312013) and the Medical Research Foundation of Guangdong province (no. B2014395). 


\section{References}

1. Jemal A, Bray F, Center MM, Ferlay J, Ward E and Forman D: Global cancer statistics. CA Cancer J Clin 61: 69-90, 2011.

2. Linos E, Spanos D, Rosner BA, et al: Effects of reproductive and demographic changes on breast cancer incidence in China: A modeling analysis. J Natl Cancer Inst 100: 1352-1360, 2008.

3. Zhang X, Li X, Zhang N, Yang Q and Moran MS: Low doses ionizing radiation enhances the invasiveness of breast cancer cells by inducing epithelial-mesenchymal transition. Biochem Biophys Res Commun 412: 188-192, 2011.

4. Kalluri R and Weinberg RA: The basics of epithelial-mesenchymal transition. J Clin Invest 119: 1420-1428, 2009.

5. Kalluri R: EMT: When epithelial cells decide to become mesenchymal-like cells. J Clin Invest 119: 1417-1419, 2009.

6. Emadi Baygi M, Soheili ZS, Schmitz I, et al: Snail regulates cell survival and inhibits cellular senescence in human metastatic prostate cancer cell lines. Cell Biol Toxicol 26: 553-567, 2010.

7. Wang J,Kuiatse I,Lee AV, et al: Sustained c-Jun-NH2-kinase activity promotes epithelial-mesenchymal transition, invasion and survival of breast cancer cells by regulating extracellular signal-regulated kinase activation. Mol Cancer Res 8: 266-277, 2010.

8. Tryndyak VP, Beland FA and Pogribny IP: E-cadherin transcriptional down-regulation by epigenetic and microRNA-200 family alterations is related to mesenchymal and drug-resistant phenotypes in human breast cancer cells. Int J Cancer 126: 2575-2583, 2010

9. Thiery JP and Sleeman JP: Complex networks orchestrate epithelial-mesenchymal transitions. Nat Rev Mol Cell Biol 7: $131-142,2006$

10. Zavadil $\mathrm{J}$ and Böttinger EP: TGF-beta and epithelial-to-mesenchymal transitions. Oncogene 29: 5764-5774, 2005.

11. Min C, Eddy SF, Sherr DH and Sonenshein GE: NF-kappaB and epithelial to mesenchymal transition of cancer. J Cell Biochem 104: 733-744, 2008.

12. Huber MA, Beug H and Wirth T: Epithelial-mesenchymal transition: NF-kappaB takes center stage. Cell Cycle 3: 1477-1480, 2004.

13. Huber MA, Azoitei N, Baumann B, et al: NF-kappaB is essential for epithelial-mesenchymal transition and metastasis in a model of breast cancer progression. J Clin Invest 114: 569-581, 2004.

14. Hugo H, Ackland ML, Blick T, Lawrence MG, Clements JA, Williams ED and Thompson EW: Epithelial-mesenchymal and mesenchymal-epithelial transitions in carcinoma progression. J Cell Physiol 213: 374-383, 2007.

15. Cai J, Parr C, Watkins G, Jiang WG and Boulton M: Decreased pigment epithelium-derived factor expression in human breast cancer progression. Clin Cancer Res 12: 3510-3517, 2006.

16. Zhou D, Cheng SQ, Ji HF, et al: Evaluation of protein pigment epithelium-derived factor (PEDF) and microvessel density (MVD) as prognostic indicators in breast cancer. J Cancer Res Clin Oncol 136: 1719-1727, 2010.

17. Yasui N,Mori T,Morito D, et al: Dual-site recognition of different extracellular matrix components by antiangiogenic/neurotrophic serpin, PEDF. Biochemistry 42: 3160-3167, 2003.

18. Aurora AB, Biyashev D, Mirochnik Y, et al: NF-kappaB balances vascular regression and angiogenesis via chromatin remodeling and NFAT displacement. Blood 116: 475-484, 2010

19. Zaichuk TA, Shroff EH, Emmanuel R, et al: Nuclear factor of activated $\mathrm{T}$ cells balances angiogenesis activation and inhibition. J Exp Med 199: 1513-1522, 2004.

20. Hoshina D, Abe R, Yamagishi SI and Shimizu H: The role of PEDF in tumor growth and metastasis. Curr Mol Med 10: 292-295, 2010

21. Fitzgerald DP, Subramanian P, Deshpande M, et al: Opposing effects of pigment epithelium-derived factor on breast cancer cell versus neuronal survival: Implication for brain metastasis and metastasis-induced brain damage. Cancer Res 72: 144-153, 2012.

22. Manalo KB and Choong PF, Dass CR: Pigment epithelium-derived factor as an impending therapeutic agent against vascular epithelial growth factor-driven tumor-angiogenesis. Mol Carcinog 50: 67-72, 2011

23. World Health Organization: World Health Organization family of international classifications. The meeting for heads of WHO collaborating centres for the family of internation classifications. Cologne, Germany, October 19-25, 2003.

24. Sobin LH and Wittekind C: TNM Classification of Malignant Tumors. Fifth edition. Wiley-Liss, New York, NY, 1997.

25. Elston EW and Ellis IO: Method for grading breast cancer. J Clin Pathol 46: 189-190, 1993
26. Remmele W and Stegner HE: Recommendation for uniform definition of an immunoreactive score (IRS) for immunohistochemical estrogen receptor detection (ER-ICA) in breast cancer tissue. Pathologe 8: 138-140, 1987 (In German).

27. Zhang M, Liu NY, Wang XE, et al: Activin B promotes epithelial wound healing in vivo through Rhoa-JNK signaling pathway. PLoS One 6: e25143, 2011.

28. Haybittle JL, Blamey RW, Elston CW, et al: A prognostic index in primary breast cancer. Br J Cancer 45: 361-366, 1982

29. Filleur S, Nelius T, de Riese $W$ and Kennedy RC: Characterization of PEDF: A multi-functional serpin family protein. J Cell Biochem 106: 769-775, 2009.

30. Setten GV and Abdiu O: Possible role of pigment epithelium derived factor in angiogenesis. European Opthalmic Rev 10: 64-67, 2009

31. Kawaguchi T, Yamagishi SI and Sata M: Structure-function relationships of PEDF. Curr Mol Med 10: 302-311, 2010.

32. Broadhead ML, Dass CR and Choong PF: Cancer cell apoptotic pathways mediated by PEDF: Prospects for therapy. Trends Mol Med 15: 461-467, 2009.

33. Jan R, Huang $M$ and Lewis-Wambi J: Loss of pigment epithelium-derived factor: A novel mechanism for the development of endocrine resistance in breast cancer. Breast Cancer Res 14: R146, 2012.

34. Uehara H, Miyamoto M, Kato K, et al: Expression of pigment epithelium-derived factor decreases liver metastasis and correlates with favorable prognosis for patients with ductal pancreatic adenocarcinoma. Cancer Res 64: 3533-3537, 2004.

35. Guan M, Yam HF, Su B, et al: Loss of pigment epithelium derived factor expression in glioma progression. J Clin Pathol 56: 277-282, 2003.

36. Cheung LW, Au SC, Cheung AN, et al: Pigment epithelium-derived factor is estrogen sensitive and inhibits the growth of human ovarian cancer and ovarian surface epithelial cells. Endocrinology 147: 4179-4191, 2006.

37. Ning Q, Liu C, Hou L, et al: Vascular endothelial growth factor receptor-1 activation promotes migration and invasion of breast cancer cells through epithelial-mesenchymal transition. PLoS One 8: e65217, 2013.

38. Seki R, Yamagishi S, Matsui $\mathrm{T}$, et al: Pigment epithelium-derived factor (PEDF) inhibits survival and proliferation of VEGF-exposed multiple myeloma cells through its anti-oxidative properties. Biochem Biophys Res Commun 431: 693-697, 2013

39. Yang J, Chen S, Huang X, et al: Growth suppression of cervical carcinoma by pigment epithelium-derived factor via anti-angiogenesis. Cancer Biol Ther 9: 967-974, 2010.

40. Zhang Y, Han J, Yang X, et al: Pigment epithelium-derived factor inhibits angiogenesis and growth of gastric carcinoma by down-regulation of VEGF. Oncol Rep 26: 681-686, 2011.

41. Cai J, Jiang WG, Grant MB and Boulton M: Pigment epithelium-derived factor inhibits angiogenesis via regulated intracellular proteolysis of vascular endothelial growth factor receptor 1. J Biol Chem 281: 3604-3613, 2006.

42. Kokkinos MI, Wafai R, Wong MK, et al: Vimentin and epithelial-mesenchymal transition in human breast cancer-observations in vitro and in vivo. Cells Tissues Organs 185: 191-203, 2007.

43. Lee J, Hahm ER, Marcus AI and Singh SV: Withaferin A inhibits experimental epithelial-mesenchymal transition in MCF-10A cells and suppresses vimentin protein level in vivo in breast tumors. Mol Carcinog 30: 10, 2013.

44. Blanco MJ, Moreno-Bueno G, Sarrio D, et al: Correlation of Snail expression with histological grade and lymph node status in breast carcinomas. Oncogene 21: 3241-3246, 2002.

45. Luo WR, Li SY, Cai LM and Yao KT: High expression of nuclear Snail, but not cytoplasmic staining, predicts poor survival in nasopharyngeal carcinoma. Ann Surg Oncol 19: 2971-2979, 2012.

46. Alkatout I, Wiedermann M, Bauer M, et al: Transcription factors associated with epithelial-mesenchymal transition and cancer stem cells in the tumor centre and margin of invasive breast cancer. Exp Mol Pathol 94: 168-173, 2013.

47. Peinado H, Olmeda D and Cano A: Snail, Zeb and bHLH factors in tumour progression: An alliance against the epithelial phenotype? Nat Rev Cancer 7: 415-428, 2007.

48. Hirsch J, Johnson CL, Nelius T, Kennedy R, Riese WD and Filleur S: PEDF inhibits IL8 production in prostate cancer cells through PEDF receptor/phospholipase A2 and regulation of NFאB and PPAR $\gamma$. Cytokine 55: 202-210, 2011. 